\title{
Instability in lateral dynamics of a metal strip in cold rolling
}

\author{
T. Tarnopolskaya* $\quad$ D. J. Gates* $\quad$ F. R. de Hoog* \\ W. Y. D. Yuen ${ }^{\dagger}$
}

(Received 28 October 2004; revised 14 September 2005)

\begin{abstract}
Consider the lateral dynamics of a strip between the uncoiler and the first stand of a cold rolling mill: operational problems are experienced in the metal rolling industry. Here we introduce a physically based model for the buckling of the strip between the uncoiler and the reduction mill. The model reveals, for the first time, that there exists a critical level of asymmetry in rolling conditions above which the strip motion becomes unstable. Below this level, two solutions of the steady-state model exist. Whereas one of these solutions represents a stable equilibrium, the other exhibits the features of unstable equilibrium. The motion of the strip is sensitive to the initial lateral
\end{abstract}

${ }^{*}$ CSIRO Mathematical \& Information Sciences, Australia.

mailto: tanya.tarnopolskaya@csiro.au

$\dagger$ BlueScope Steel Research, Australia.

See http://anziamj.austms.org.au/V46/CTAC2004/Tarn for this article, (C) Austral. Mathematical Soc. 2005. Published October 7, 2005. ISSN 1446-8735 
deviation of the strip. For a given level of asymmetry, a sudden transition to unstable motion occurs if a critical lateral deviation is reached. The model can be used as a basis for development of a multi-stand model of lateral dynamics.

\section{Contents}

1 Introduction

C988

2 Mathematical model

C990

2.1 Mill model . . . . . . . . . . . . . . . . . . . . . . . C990

2.2 Strip buckling . . . . . . . . . . . . . . . . . . . . C C991

2.3 Strip dynamics . . . . . . . . . . . . . . . . . . . C992

2.4 Steady-state solution for strip off-center . . . . . . . . C995

3 Numerical examples and discussion

C995

3.1 Steady-state solution for a strip constrained upstream . . . C996

3.2 Dynamics of a strip constrained upstream . . . . . . . . C998

References

C998

\section{Introduction}

The sudden deviation of a strip from the direction of rolling, known as strip track-off, is a serious operational problem in metal rolling that could lead to catastrophic consequences, such as strip chew-up and mill crash. The strip tracking failure occurs suddenly, which poses difficulties for experimental study. A continued trend towards increased speed of the rolling process aggravates a tendency for strip track-off. The strip tracking problem has attracted considerable interest over the last decade $[1,2,3,4,5,6]$. While the 
causes of instability in the lateral dynamics of a so-called 'tail out' scenario (when the unconstrained end of the strip moves through the reduction rolls) are well understood [1], the causes of lateral instability during the rolling of a strip constrained upstream in the tension producing device or uncoiler have not been explained to date.

This paper continues our earlier study [1] where the steady-state motion of the strip constrained upstream in the uncoiler was investigated. It was revealed that the lateral motion is stable if no buckling occurs. We further argued, however, that the buckling of the strip could lead to increased lateral deviations and potential instability. The importance of strip buckling has been acknowledged by Matsumoto and Ishii [3] who found that experiments could not be explained without the introduction of an adjustable parameter to make allowance for the strip buckling effect in their model.

This paper studies the effect of strip buckling on lateral dynamics of the strip constrained upstream in the uncoiler. This is a first attempt to introduce a physically based strip buckling model into the model of lateral dynamics. Our analysis of strip buckling between the uncoiler and the reduction stand is based on a simplified strip buckling model suggested by Benson [7].

The developed model reveals, for the first time, that the buckling of the strip changes the nature of the lateral dynamics of the strip. While, in the absence of strip buckling, the lateral dynamics is inherently stable [1], the buckling of the strip caused by the bending moment due to the asymmetry in rolling conditions could lead to instability. We found that for a given type of asymmetry in the rolling conditions, there exists a critical level of asymmetry above which the lateral motion becomes unstable. Below this level, two steady-state solutions are possible: one is associated with a small amount of buckling and represents a stable motion, while the other is associated with a significant amount of buckling and exhibits the features of unstable equilibrium. For a given level of asymmetry, there is a critical strip deviation above which the motion becomes unstable. 


\section{Mathematical model}

In this section the constitutive parts of the model of lateral dynamics of the strip in a single stand of a cold rolling mill are briefly reviewed.

\subsection{Mill model}

A model of thickness reduction of the strip in a cold rolling mill has been described in a number of papers $[8,9,10,11,12]$. This model is rather complicated and we do not discuss it in detail here. The model consists of coupled models of the plastic deformation of the strip in the roll bite and elastic deformation of the roll stack for non-symmetric loading and mill geometry. The model of strip deformation in the roll bite is coupled with the model of the deformation and motion of the strip outside the roll bite through the boundary conditions at the interface between two regions, which can be formulated in terms of strip velocity and longitudinal tensile stresses in the strip. For the purpose of the subsequent lateral dynamics analysis, it is convenient to express the boundary conditions in terms of the in-plane rotational speed of the strip $\omega_{i}$ and the in-plane bending moment $M_{i}$ in the form

$$
f_{i}\left(\omega_{i}, M_{i}\right)=0, \quad i=1,2,
$$

where indices $i=1,2$ refer to the entry and exit of the roll bite,

$$
\begin{aligned}
\omega_{i} & =\tilde{v}_{i}(x) / x, \\
M_{i} & =\int_{-W / 2}^{W / 2} \sigma_{i}(x) x d x,
\end{aligned}
$$

$\tilde{v}_{1}(x)$ and $\tilde{v}_{2}(x)$ are the deviations of the velocities of the strip from their mean values (assumed to be linear functions of $x$ ), $x$ is the co-ordinate across the strip width measured from the center of the strip, $\sigma(x)$ is the tensile stress distribution in the strip, and $W$ is the strip width. 


\subsection{Strip buckling}

A simplified model of strip buckling proposed by Benson [7] is based on stress distribution in an Euler beam and the assumptions that the onset of buckling occurs at an infinitesimally small negative stress. It is also assumed [7] that the stress distribution in the strip after buckling takes place remains linear. According to Benson's model, the tensile stress distribution across the strip width at a given position along the strip axis after the buckling takes place is

$$
\sigma_{m}=\max \left(0, b_{1}+b_{2} x\right),
$$

where $b_{1}$ and $b_{2}$ are determined by the equations

$$
\begin{gathered}
\int_{-W / 2}^{W / 2} \sigma_{m} d x=T \\
\int_{-W / 2}^{W / 2} \sigma_{m} x d x=M .
\end{gathered}
$$

Here $M$ and $T$ are the in-plane moment and the tensile force acting on the cross-section of the strip. They are assumed to be known.

Benson [7] interprets the bending of the strip under buckling in terms of the bending of the Euler beam with reduced effective width and bending moment, which allows a simplified treatment of the buckled strip on the basis of Euler beam theory for the purpose of deflection analysis.

On the basis of Benson's model, it can be shown that the in-plane curvature of the strip after the buckling occurs is

$$
\kappa_{B}(\bar{M}, T)=\frac{T W}{6 E I} \times \begin{cases}\bar{M}, & \text { if }|\bar{M}| \leq 1 ; \\ \frac{4}{(3-\bar{M})^{2}}, & \text { if } \bar{M}>1 ; \\ -\frac{4}{(3+\bar{M})^{2}}, & \text { if } \bar{M}<-1 ;\end{cases}
$$


where

$$
\bar{M}=\frac{6 M}{T W}, \quad-3<\bar{M}<3,
$$

$I$ is the moment of inertia of the cross-section, and $E$ is Young modulus.

\section{$2.3 \quad$ Strip dynamics}

The equations of strip motion can be written in the form [3]

$$
\begin{aligned}
\frac{d \theta_{1}}{d t} & =\omega_{1}\left(D_{1}, M_{1}\right)+\bar{v}_{1} \kappa_{1}^{+}, \\
\frac{d D_{1}}{d t} & =\bar{v}_{1} \theta_{1},
\end{aligned}
$$

where $\theta_{1}$ is the angle the strip centerline forms with the rolling direction, $D_{1}$ is the lateral deviation of the strip centerline, $M_{1}$ is the moment acting on the strip, $\kappa_{1}^{+}$is the in-plane curvature of the strip at the entry of the roll bite, $\bar{v}_{1}$ is the mean speed, and $t$ is the time. All above quantities are taken at the entry of the roll bite. In what follows, we distinguish between the strip curvature at the entry of the roll bite $\kappa_{1}^{+}$and the strip curvature upstream adjacent to the entry of the roll bite $\kappa_{1}^{-}$. In doing this, we recognise that no buckling occurs at the entry of the roll bite where the strip is compressed between the rolls. However, a significant buckling could develop near the entry due to the bending moment applied to the strip at the entry of the roll bite. A detailed discussion of the shape of the buckling eigenmode will be given elsewhere. Here, for the sake of simplicity, we assume that the curvature adjacent to the entry of the roll bite upstream is given by Benson's model

$$
\kappa_{1}^{-}=\left.\kappa_{B}(M, T)\right|_{M=-M_{1}} .
$$

At the entry of the roll bite, the entry curvature of the strip is

$$
\kappa_{1}^{+}=-\frac{M_{1}}{E I} .
$$


Obviously, if no strip buckling occurs,

$$
\kappa_{1}^{+}=\kappa_{1}^{-} .
$$

Equations $(9,10)$ contain 3 unknowns, namely $\theta_{1}, D_{1}$ and $M_{1}$, and therefore an additional equation is required to obtain a complete system of equations for strip lateral dynamics. An additional equation can be derived from the strip bending model.

Benson's buckling model suggests that the strip can be treated in a simplified manner as an Euler beam with varying effective width. A model of strip lateral dynamics with strip deflections described by Benson's model will be presented elsewhere. In this paper, for the sake of simplicity, we treat strip deflections using the Euler beam model with constant effective width. The effective width can be chosen so as to minimise the inaccuracy introduced by such a simplification. In what follows, the effective width is calculated at the entry of the roll bite. Such a simplified model retains important features of the complete model and provides insight into new features of the lateral dynamics of the strip introduced through the strip buckling model.

It can be shown that the deflections of the strip with constant effective width calculated from Benson's model at the entry of the roll bite is described by the equation

$$
\frac{\partial^{2} u}{\partial z^{2}}=\kappa_{B}\left(-M_{1}, T\right)+\frac{G}{E I_{\mathrm{eff}}}(L-z)-\frac{T}{E I_{\mathrm{eff}}}\left(\left.u\right|_{z=L}-u\right),
$$

where $u$ is the lateral deflection of the strip centerline, $I_{\text {eff }}$ is the moment of inertia of the cross-section with reduced effective width, $z$ is the co-ordinate along the strip centerline, with origin at a distance $L$ upstream of the roll bite, and $G$ is the lateral force. It can be shown that the last term on the right hand side of (14) is negligible for the range of tensile forces of interest in cold rolling. 
The boundary conditions at the uncoiler, at the distance $L$ upstream from the reduction roll are taken in the form

$$
\left.u\right|_{z=0}=\left.\frac{\partial u}{\partial z}\right|_{z=0}=0 .
$$

It can be shown that the solution of equations $(14,15)$ can be represented in the form

$$
\begin{gathered}
D_{1}=\left.u\right|_{z=L}=L^{2}\left[\frac{G L}{3 E I_{\mathrm{eff}}}+\frac{\kappa_{B}\left(-M_{1}, T\right)}{2}\right], \\
\theta_{1}=-\left.\frac{\partial u}{\partial z}\right|_{z=L}=L\left[-\kappa_{B}\left(-M_{1}, T\right)-\frac{L G}{2 E I_{\mathrm{eff}}}\right] .
\end{gathered}
$$

Eliminating the lateral force $G$ from equations $(16,17)$ yields the following expression for the entry moment as a function of the lateral entry deviation and entry angle

$$
-\kappa_{B}\left(-M_{1}, T\right)=\frac{2}{L^{2}}\left(3 D_{1}+2 \theta_{1} L\right) .
$$

Differentiating equation (18) with respect to time $t$ and making use of equations $(9,10)$ yield the following system of ordinary differential equations that describes the lateral dynamics of the strip

$$
\begin{aligned}
\frac{d D_{1}}{d t} & =-\bar{v}_{1}\left[\frac{\kappa_{B}\left(-M_{1}, T\right) L}{4}+\frac{3 D_{1}}{2 L}\right] \\
\frac{d \theta_{1}}{d t} & =\omega_{1}\left(D_{1}, M_{1}\right)-\bar{v}_{1} \frac{M_{1}}{E I} \\
\frac{d M_{1}}{d t} & =-\left(\frac{\partial \kappa_{B}}{\partial M_{1}}\right)^{-1} \frac{2}{L^{2}}\left(3 \bar{v}_{1} \theta_{1}+2 L \omega_{1}\left(D_{1}, M_{1}\right)-2 L \bar{v}_{1} \frac{M_{1}}{E I}\right) .
\end{aligned}
$$

The system (19-21) is solved subject to the initial conditions

$$
\left.\theta_{1}\right|_{t=0}=\theta_{1}^{0} ;\left.\quad D_{1}\right|_{t=0}=D_{1}^{0} ;\left.\quad M_{1}\right|_{t=0}=M_{1}^{0} .
$$


Obtain the functional relationship $\omega_{1}\left(D_{1}, M_{1}\right)$ from the rolling model [1, 2 ], for given boundary conditions at the exit of the roll bite and the level of asymmetry in the rolling conditions, by interpolating the results of the numerical simulations. For all cases considered in this paper, the linear function of the form $\omega_{1}\left(D_{1}, M_{1}\right)=\alpha_{0}+\alpha_{1} D_{1}+\alpha_{2} M_{1}$ provided sufficient accuracy.

\subsection{Steady-state solution for strip off-center}

At steady-state, the equations of strip motion (19-21) reduce to

$$
\begin{aligned}
\theta_{1} & =0 \\
\omega_{1}\left(D_{1}, M_{1}\right)+\bar{v}_{1} \kappa_{1}^{+}\left(M_{1}\right) & =0 \\
6 D_{1}+\kappa_{B}\left(-M_{1}, T\right) L^{2} & =0
\end{aligned}
$$

where $\omega_{1}=\omega_{1}\left(D_{1}, M_{1}\right)$ is calculated from the rolling model $[1,2]$ for a given asymmetry and mill schedule.

\section{Numerical examples and discussion}

In this section we study the steady-state lateral motion and dynamics of the strip constrained upstream in the uncoiler at a given distance from the reduction rolls. The data used for the calculations is as in [1]. The asymmetry in rolling conditions is represented by the unequal roll forces acting on the sides of the rolls. The level of asymmetry is defined as the ratio of roll forces on the right and left sides of the rolls. 
(a)

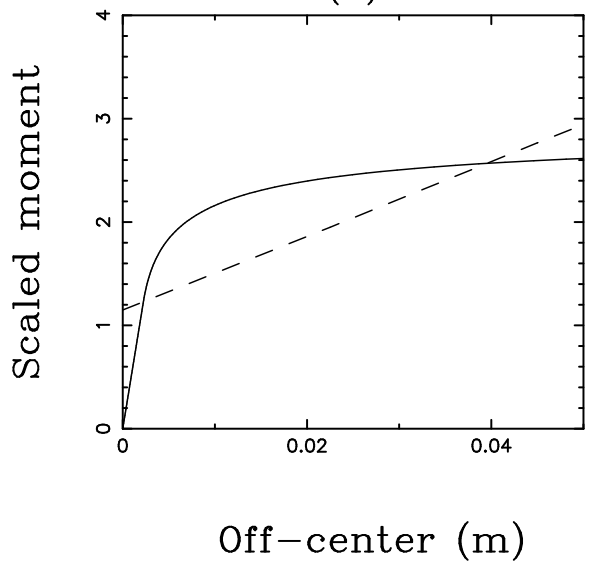

(b)

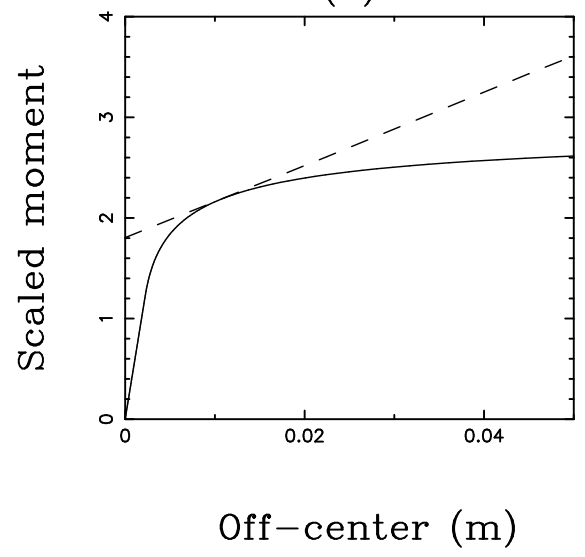

Figure 1: Graphical solution for a steady-state strip off-center $\left(D_{1}\right)$ under the ratio of roll forces on the right and left sides of the rolls of (a) 0.85 ; (b) 0.8. Scaled moment is defined by equation (8).

\subsection{Steady-state solution for a strip constrained upstream}

The graphical illustration of the steady-state solution is shown in Figure 1, where the solid line shows the solution of equations $(23,25)$, while the dashed line shows the solution of equation (24). The steady-state lateral deviation of the strip at the entry of the roll bite is given by the intersection of these two curves. Figure 1a reveals that for a smaller level of the asymmetry, two steady-state solutions are possible. The smaller one corresponds to a small amount of buckling, while the larger corresponds to a significant amount of buckling. While the smaller solution increases with increase in the level of asymmetry, the larger solution decreases with increase in the level of asymmetry. The two solutions converge to a single solution at a certain critical level of asymmetry (Figure 1b). Above the critical level of asymmetry, no 


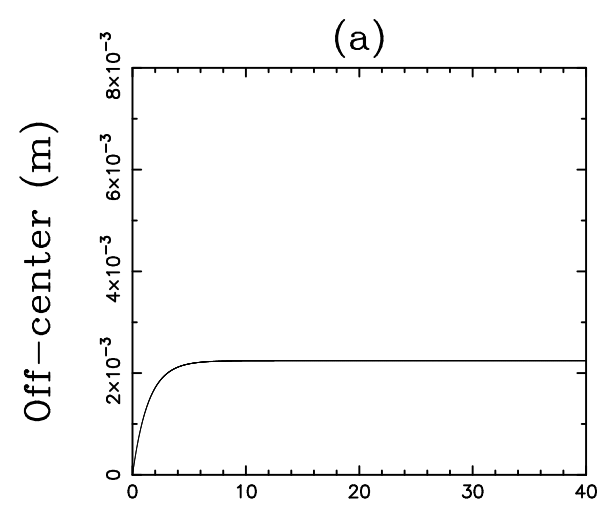

Time ( $)$

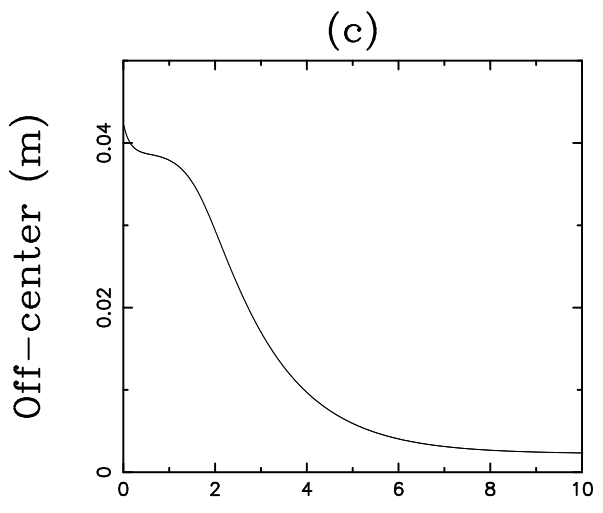

Time (s)

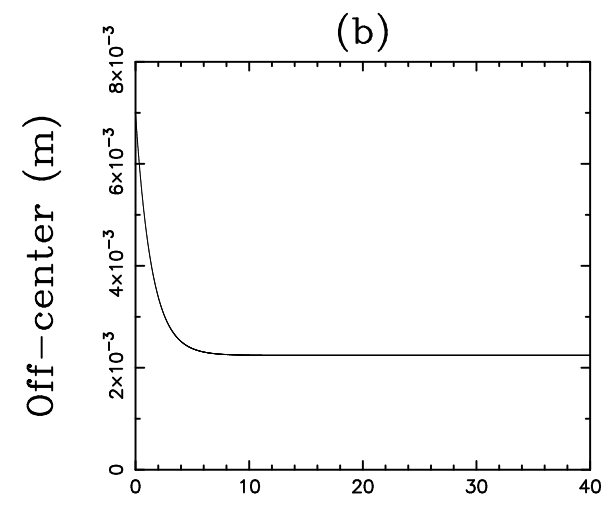

Time ( $)$

(d)

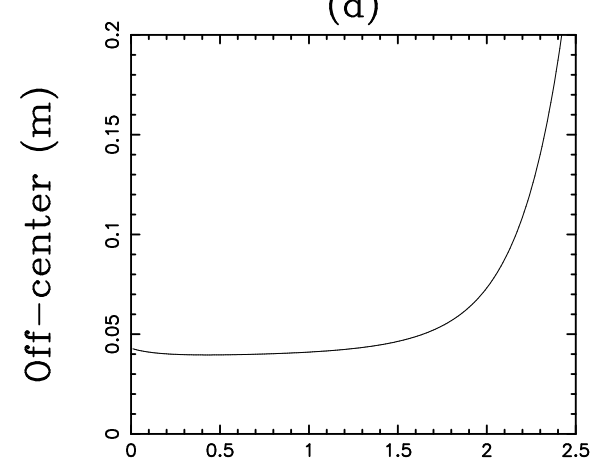

Time ( $)$

Figure 2: Strip lateral dynamics for rolling conditions as in Figure 1a and different initial strip off-center: (a) $D_{1}^{0}=0$; (b) $D_{1}^{0}=7 \mathrm{~mm}$; (c) $D_{1}^{0}=$ $42.5 \mathrm{~mm}$; (d) $D_{1}^{0}=43 \mathrm{~mm}$. 
steady-state solution exists. Thus, the lateral motion becomes unstable once a critical level of asymmetry is reached, which may explain a sudden onset of instability observed in practice. The critical level of asymmetry is a function of the mill schedule parameters.

\subsection{Dynamics of a strip constrained upstream}

Examples of the lateral dynamics of the strip, in terms of the strip lateral deviation at the entry of the roll bite as a function of time, are shown in Figure 2 for the asymmetry level corresponding to Figure 1a. See that the lateral dynamics of the strip is sensitive to the strip initial position. There is a sudden transition from stable to unstable lateral motion when a certain value of the initial strip lateral deviation, $D_{1}^{0}$, is reached. This value depends on the level of asymmetry and, as numerical simulations suggest, is close to the larger solution of the steady-state problem for a given level of asymmetry. Figure 2 also suggests that while the smaller solution of the steady-state problem represents a stable equilibrium, the larger solution exhibits the features of an unstable equilibrium.

Acknowledgments: we thank the management of BlueScope Steel Research for permission to publish the information contained in this paper.

\section{References}

[1] T. Tarnopolskaya, D. Gates, F. R. de Hoog, W. Y. D. Yuen and A. Dixon, Numerical analysis of lateral movement of a metal strip during cold rolling, ANZIAM J., volume 45(E), pages C173-C186, 2004. http://anziamj.austms.org.au/V45/CTAC2003/Tarn C988, C989, C995 
[2] T. Tarnopolskaya, F. R. de Hoog, D. J. Gates, A. Dixon and W. Y. D. Yuen, Analysis of strip track-off during flat rolling, Proceedings of 8th International Rolling Conference, volume XL, Florida, pages 237-246, 2002. C988, C995

[3] H. Matsumoto and A. Ishii, Three-dimensional simulation of snake motion in a tandem cold mill, Iron $\&$ Steel Maker, vol. 30(12), pages 31-39, 2003. C988, C989, C992

[4] T. Ishikawa, Y. Tozava and J. Nishizawa, Fundamental study on snaking in strip rolling, Transactions ISIJ, vol. 28, pages 485-500, 1988. C988

[5] A. Nilson, FE simulations of camber in hot strip rolling, Journal of material processing technology, vol. 80-81, pages 325-329, 1998. http://dx.doi.org/10.1016/S0924-0136(98)00144-7 C988

[6] D. L. Biggs, S. J. Hardy and K. J. Brown, Finite element modelling of camber development during hot rolling of strip steel, Ironmaking and Steelmaking, vol. 25(1), pages 81-89, 1998. C988

[7] R. C. Benson, Lateral dynamics of a moving web with geometrical inperfections, Journal of dynamical systems, Measurement and Control, volume 124, pages 25-34, 2002. http://dx.doi.org/10.1115/1.1435643 C989, C991

[8] H. Matsumoto and T. Kawanami, Mechanism of material deformation related to shape and crown phenomena, Proceedings 4 th International Steel Rolling Conference, E6.1-E6.11, 1987. C990

[9] H. Matsumoto and K. Yamada, Generalised 2-dimensional theory of flat rolling and comparison with 3-dimensional FEM, Advanced Technology of Plasticity, Proc. of 5th ICTP, vol. I, pages 3-6, 1996. C990 
[10] W. J. Edwards and P. D. Spooner, Analysis of strip shape. In: Automation of tandem mills, G. F. Bryant (Ed.), pages 176-212, 1973. C990

[11] T. Tarnopolskaya and F. de Hoog, An efficient method for strip flatness analysis in cold rolling, Mathematical Engineering in Industry, 7(1), pages 71-95, 1998. C990

[12] W. Y. D. Yuen, A. Duwal and B. Wechner, A new formulation for the analysis of strip flatness in strip rolling, Proceedings 7th International Conference on Steel Rolling, pages 296-301,1998. C990 